\title{
Aplicación de la metodología FPEIR al diagnóstico ambiental del Humedal Lucre-Huacarpay, 2017
}

Yeny Acostupa ${ }^{\mathbf{1 , a}}$, Diana Aréstegui ${ }^{\mathbf{2}, \mathbf{b}}$, Edilberto Castro ${ }^{\mathbf{3}, \mathbf{c}}$, Walter Choquevilca ${ }^{\mathbf{4 , d}}$, Giovana Guzmán”,e, Paul Sánchez ${ }^{6, c}$.

1. Universidad San Antonio Abad del Cusco. Cusco, Perú

2. Universidad Andina del Cusco. Cusco, Perú

3. Consultor independiente. Cusco, Perú

4. Consultor independiente. Cusco, Perú

5. Universidad San Antonio Abad del Cusco. Cusco, Perú

6. Consultor independiente. Cusco, Perú

a. Físico-matemático. M.Sc. Docente Investigadora, b. Lic. en Turismo. M.Sc. Docente Investigadora, c. Biólogo Investigador. M.Sc, d. Ing. Zootecnista Investigador, e. Físico. M.Sc. Docente Investigadora, F.

M.Sc. Consultor independiente

Distrito de Lucre, provincia de Quispicanchis, Región Cusco

\section{RESUMEN}

La aplicación de la metodología FPEIR (Fuerzas Motrices, Presión, Estado, Impacto y Respuesta), evalúa la sostenibilidad del manejo del Humedal Lucre-Huacarpay, empleando indicadores de fuerzas motrices, presión, estado, impacto y respuesta; y está elaborado para que sirva como base para una posterior investigación acerca del diagnóstico ambiental sobre el humedal.

\section{Objetivos}

Realizar un diagnóstico ambiental del Humedal Lucre- Huacarpay a través de la aplicación de la metodología FPEIR.

\section{Materiales y métodos}

Se analizaron los factores biofísicos, tecnológicos, socio-económicos y políticoinstitucionales, con el fin de conocer el estado actual y la tendencia que presenta el Humedal Lucre- Huacarpay, por lo que se hizo un análisis de estudios previos, bases de datos de municipalidades y observación en campo, además de lineamientos basados en el modelo de enfoque FPEIR propuesto por la Organización para la Cooperación y el Desarrollo Económico - OCDE (1993), y el que evalúa el ambiente y los recursos naturales por las presiones socio-económicas directas e indirectas sobre el patrimonio natural y sus impactos 
por la eliminación de desechos sólidos, líquidos y emisiones sobre el medio ambiente. Mediante este modelo pudimos identificar la situación actual del ambiente y, para entender cómo tiene lugar el proceso de degradación del Humedal Lucre-Huacarpay. Esta investigación se realizó con observaciones in situ y revisión de información secundaria, los cuales registraron 33 indicadores para la construcción del «Índice de Sostenibilidad del Humedal Lucre-Huacarpay» (ISHLH), de los cuales: 4 corresponden a fuerzas motrices, 8 son de presión, 8 de estado, 4 de impacto y 9 de respuesta.

\section{Resultados}

Las fuerzas motrices representan el $12 \%$ de los indicadores encontrados en el estudio entre los que resaltan con mayor valor son: el crecimiento demográfico y el incremento de las actividades económicas. Por otro lado, los indicadores de presión hacen un $24 \%$ del total, con los indicadores: emisión de gases de efecto invernadero y las actividades turísticas alrededor de la laguna de Huacarpay. En cuanto a los indicadores de estado, se tiene que forman parte del $24 \%$ del total de indicadores encontrados; de estos, los más resaltantes son: la calidad del agua y los conflictos por el uso de la laguna de Huacarpay. Con referencia a los indicadores de impacto, tenemos: $12 \%$ del porcentaje total de los mismos, con pérdida de biodiversidad y disminución de la calidad del agua del humedal. En los indicadores de respuesta, se obtuvieron dos que tienen mayor puntuación que son: el reconocimiento del Humedal Lucre-Huacarpay como sitio Ramsar (Lista de Humedales de Importancia Internacional) y la aplicación de tecnologías para la conservación del Humedal. Ambos inmersos en el $28 \%$ del total de indicadores encontrados en toda la metodología FMPEIR.

Conclusiones. Los resultados de la aplicación del índice, mostraron una sostenibilidad media-baja del humedal y, a partir de la respuesta, se pueden diseñar estrategias de sostenibilidad en torno al complejo del humedal.

Palabras clave: Sostenibilidad, diagnóstico, humedal, manejo. 


\section{ABSTRACT}

Objectives: To carry out an environmental diagnosis of the Humedal Lucre-Huacarpay through the application of the DPSIR methodology. Materials and methods. The biophysical, technological, socio-economic and political-institutional factors were analyzed to know the current state and the trend presented by the Lucre-Huacarpay Wetland. For that, an analysis of previous studies, databases of municipalities and observation in the field as well as guidelines based on the DPSIR Approach Model proposed by the Organization for Economic Cooperation and Development (OECD) (1993) and the one that evaluates The environment and natural resources due to direct and indirect socio-economic pressures on the natural heritage and its impacts on the disposal of solid wastes, liquids and emissions to the environment. Through this model we were able to identify the current situation of the environment and to understand how the process of degradation of the Lucre-Huacarpay wetland that took place. This research was carried out with in situ observations and secondary information review, which registered 33 indicators for the construction of the Sustainability Index of the Lucre-Huacarpay Wetland (ISHLH), of which 4 correspond to driving forces, 8 are pressure, 8 for State, 4 for Impact and 9 for Response. Results. The driving forces represent $12 \%$ of the indicators found in the study among those that stand out with greater value are the demographic growth and the increase of the economic activities. On the other hand, the pressure indicators make up $24 \%$ of the total, with indicators emitting greenhouse gases and tourism activities around the Huacarpay lagoon. As for the state indicators, they are part of $24 \%$ of the total indicators found; of these, the most noteworthy are the water quality and conflicts over the use of the Huacarpay wetland. In terms of impact indicators, there is $12 \%$ of the total percentage of the indicators, with the indicators loss of biodiversity and decrease of the water quality of the wetland. In the response indicators, two that have the highest score are the recognition of the Lucre-Huacarpay wetland as a Ramsar Site. Both immersed in $28 \%$ of the total indicators found throughout the FMPEIR methodology. Conclusions. The results of the application of the index showed a medium-low sustainability of the wetland and from the response can be designed sustainability strategies around the complex of the wetland.

Key words: Sustainability, diagnosis, wetland, management. 


\section{INTRODUCCIÓN}

El Humedal Lucre-Huacarpay en la actualidad, tiene diversos problemas ambientales causados por los mismos habitantes, los visitantes y las actividades económicas que están relacionadas al aprovechamiento de los recursos en el humedal. El ámbito del actual Humedal Lucre - Huacarpay, pertenece a la Provincia de Quispicanchis en la Región Cusco; a $25 \mathrm{~km}$ de la ciudad del Cusco, a $3020 \mathrm{msnm}$. Este humedal está constituido por tres lagunas principales: Huatón, Huacarpay, Lucre y, cuatro pantanos Huáscar, Uncca, Anchibambay y Huacarpay, que constituyen un conjunto fragmentado de lagunas, pantanos y áreas circundantes por actividades naturales y antrópicas.

Por el norte limita con los distritos de: Oropesa, San Salvador y Caicay; por el sur con la Provincia de Paruro y Distrito de Rondocan; por el este con el Distrito de Andahuaylillas y por el oeste con el Distrito de San Jerónimo. En cuanto a la denominación, el Humedal Lucre Huacarpay (1979 Ha) posee la denominación de sitio Ramsar, a partir del 23 de septiembre de 2006, la Convención sobre los Humedales (Ramsar, Irán, 1971), es el tratado intergubernamental que ofrece el marco para la conservación y el uso racional de los humedales y sus recursos. (UNESCO, 1971).

En tal sentido, mediante este trabajo se realiza la descripción de la zona de estudio y, posteriormente, se aplica la metodología FPEIR, en la que se encuentran cada uno de los indicadores que pueden servir a la población y a las instituciones públicas y privadas, con el fin de mejorar la calidad ambiental de este humedal, evaluando factores biofísicos, tecnológicos, socio-económicos y político-institucionales.

El enfoque FPEIR, sirve para entender cómo tiene lugar el proceso de degradación del ambiente. Este modelo FPEIR, afirma que las fuerzas motrices ejercen presiones sobre el medio ambiente y dichas presiones pueden provocar cambios en su estado o condición. Los impactos consecuentes sobre los atributos socioeconómicos y biofísicos provocan una respuesta de la sociedad, mediante el desarrollo o el cambio de las políticas ambientales y económicas y el desarrollo de programas destinados a prevenir, minimizar o mitigar las presiones y las fuerzas motrices. 
En este marco, el uso de indicadores que contribuyan a valorar la fragilidad del territorio y a demostrar, cuantificar y evaluar la magnitud de sus cambios puede ser de gran utilidad socioeconómica en la formulación de políticas locales y regionales, para la toma de decisiones referentes al manejo y uso sostenible del Humedal Lucre-Huacarpay.

\section{MATERIALES Y MÉTODOS}

Se realizó un estudio descriptivo en el mes de enero de 2017, donde se analizaron los factores biofísicos, tecnológicos, socio-económicos y político-institucionales, para conocer el estado actual y la tendencia que presenta el Humedal Lucre-Huacarpay. Se realizó un análisis de estudios previos, bases de datos de municipalidades y observación en campo, además, de lineamientos del modelo de enfoque FPEIR.

Este modelo está basado en el propuesto por la Organización para la Cooperación y el Desarrollo Económico - OCDE (1993) denominado Presión-Estado-Respuesta (PER). Este modelo evalúa el ambiente y los recursos naturales de las presiones socio-económicas directas e indirectas sobre el patrimonio natural y sus impactos por la eliminación de desechos sólidos, líquidos y emisiones al medio ambiente. El modelo permite identificar la situación actual del ambiente e integra en su análisis la forma en que las acciones antrópicas responden ante las presiones y tendencias sobre la calidad y degradación del ambiente (Pérez, 2003; Castro, 2002; OCDE, 1993)

El enfoque del trabajo sirve para entender cómo tiene lugar el proceso de degradación del Humedal Lucre-Huacarpay y la adopción del modelo FPEIR. Afirma que las fuerzas motrices ejercen presiones sobre el medio ambiente y dichas presiones pueden provocar cambios en su estado o condición. Los impactos consecuentes sobre los atributos socioeconómicos y biofísicos provocan una respuesta de la sociedad mediante: el desarrollo o el cambio de las políticas ambientales y económicas y, el desarrollo de programas destinados a prevenir, minimizar o mitigar las presiones y las fuerzas motrices.

Se consideró la evaluación del sistema de indicadores de fuerzas motrices, presión, estado, impacto y respuesta, basándonos en información del Instituto Nacional de Informática y Estadística (INEI) y datos estadísticos provenientes de municipalidades y ONG que 
estudiaron la zona en años anteriores y teniendo en cuenta los conceptos de los componentes del modelo FPEIR donde:

Fuerzas motrices: son las que describen las fuerzas que ejercen presión sobre el Humedal Lucre- Huacarpay. El crecimiento demográfico y el desarrollo económico son considerados como fuerzas motrices del cambio ambiental, e incluyen, aspectos particulares que ejercen presión: energía, transporte, urbanización y globalización.

Indicadores de presión: son aquellos que describen las presiones o intervenciones directas e indirectas que las actividades humanas ejercen sobre el medio natural. Los indicadores de presión se clasifican en dos grupos: el primero de ellos representa las presiones directas sobre el ambiente ocasionadas por las actividades humanas, tales como: el volumen de residuos generados, la emisión de contaminantes al aire, entre otros. En el segundo grupo, están las actividades humanas en sí mismas, es decir, las condiciones de las actividades productivas o actividades que generan la problemática (Pérez, 2003).

Los indicadores de estado, evalúan cuantitativa y cualitativamente el estado de los recursos naturales y del medio ambiente. Se refieren a la calidad del ambiente, así como a la cantidad y estado de los recursos naturales, por ejemplo, la calidad del aire (concentraciones de contaminantes) o del agua, de igual manera, a la cantidad de recursos naturales. Dichos indicadores constituyen, en general: las políticas de protección ambiental.

Los indicadores de impacto: determinan los impactos producidos por las actividades económicas sobre el estado de los recursos naturales y del medio ambiente. Dichos indicadores constituyen, generalmente, los elementos a corregir, minimizar y monitorear, para los objetivos de las políticas de protección ambiental. En este tipo de indicadores se incluyen, los efectos a la salud de la población y a los ecosistemas causados, precisamente, por el deterioro de la calidad ambiental.

Finalmente, los indicadores de respuesta: son el resultado de las presiones sobre el medio ambiente. Se generan diferentes respuestas desde el orden socio-político para evitar, corregir, mitigar o cambiar tendencias en el comportamiento de los individuos o de las políticas, que por diferentes razones, resultan lesivas al medio ambiente. Todos estos esfuerzos privados y 
públicos se incorporan a los «indicadores de respuesta». Estos indicadores presentan los esfuerzos realizados por la sociedad o por una institución para reducir o mitigar la degradación del ambiente (Pérez, 2003).

Para este estudio, se efectuó la selección de aquellos indicadores de Fuerza Motriz, Presión, Estado, Impacto y Respuesta (FPEIR), que mayor incidencia tendrían en el manejo del Humedal Lucre-Huacarpay; se realizó con ayuda de observaciones in situ y revisión de información secundaria, los cuales registraron 33 indicadores para la construcción del «Índice de Sostenibilidad del Humedal Lucre-Huacarpay» (ISHLH), de los cuales, 4 corresponden a fuerzas motrices, 8 son de presión, 8 de estado, 4 de impacto y 9 de respuesta.

\section{1.- INDICADORES DE FUERZA MOTRIZ}

\section{a) Crecimiento demográfico}

Según el censo realizado por el INEI en el año 2007, el Distrito de Lucre cuenta con 4256 habitantes, frente a los 3956 habitantes que fueron el resultado del censo del año 2000. Esto demuestra que en 7 años se ha dado un crecimiento demográfico del $7 \%$, sin embargo, al censo de 1993 la tasa de evolución poblacional era de 1,68 \%, lo que indica que el crecimiento se ha acelerado e incrementado en un 5,32 \%. Actualmente, la densidad de población calculada representa a 35,8 hab. $/ \mathrm{km}^{2}$.

\section{b) Incremento de actividades económicas}

Entre las actividades económicas que se desarrollan en el Distrito de Lucre, están la agricultura, ganadería, la caza, la silvicultura, la pesca, explotación de canteras y actividades relacionadas al turismo. Según datos de indicadores de trabajo y empleo al año 2004, la PEA (Población Económicamente Activa) de 6 y más años es de 887 individuos (718 mujeres y 169 varones) que representan al 38,7 \%. De este total, la población ocupada de 15 años a más, en actividades de agricultura (actividad principal) alcanza el $49 \%$ y, las demás actividades mencionadas representan el $51 \%$. 
Las actividades de agricultura realizadas en torno al Humedal Lucre-Huacarpay se desarrollan en 5163,29 Has.; de esta, el $61 \%$ se encuentra bajo la administración de 6 comunidades campesinas, los pequeños y medianos propietarios conducen el $37 \%$.

\section{c) Implementación de políticas públicas}

Ley General del Ambiente n. ${ }^{\circ} 28$ 611:

99.1 En el ejercicio de sus funciones, las autoridades públicas adoptan medidas de protección especial para los ecosistemas frágiles, tomando en cuenta sus características y recursos singulares; y su relación con condiciones climáticas especiales y con los desastres naturales.

99.2 Los ecosistemas frágiles comprenden, entre otros, desiertos, tierras semiáridas, montañas, pantanos, bofedales, bahías, islas pequeñas, humedales, lagunas alto andinas, lomas costeras, bosques de neblina y bosques relicto.

99.3 El Estado reconoce la importancia de los humedales como hábitat de especies de flora y fauna, en particular de aves migratorias, priorizando su conservación en relación con otros usos.

«Lograr la gestión integrada y sostenible [...]» de esos ecosistemas (Política Nacional del Ambiente. Eje 1, numeral 6). http://www.minam.gob.pe/wp-content/uploads/2013/08/Pol\%C3\%ADticaNacional-del-Ambiente.pdf

Ordenanza N. ${ }^{\circ}$ 03-2013-MDL-Q, que crea el Comité de Gestión Participativa del Sitio Ramsar Humedal Lucre-Huacarpay, el 23 de setiembre de 2013.

Plan de gestión participativa 2014. Incluye: visión, misión, un diagnóstico socio-ecológico, problemas y plan de gestión. Se hizo también un diagnóstico participativo.

Proyecto de inversión pública; «Ampliación y mejoramiento del sistema de agua potable e instalación de letrinas en la comunidad de Pacramayo, Distrito de Lucre, Provincia de Quispicanchi - Cusco», con código SNIP N. ${ }^{\circ}$ 79521, de la municipalidad, en favor del Gobierno Regional Cusco. 


\section{d) Variabilidad climática}

Según datos del año 2014, en el Humedal Lucre-Huacarpay, la temperatura media era superior a los $10^{\circ} \mathrm{C}$, y la precipitación media anual era de $656 \mathrm{~mm}$, por otro lado, el periodo de precipitación mayor a $200 \mathrm{~mm}$, alcanzaba su máximo valor en el mes de enero. Sin embargo, estudios realizados en el año 2016 demuestran que la temperatura actual es, en promedio, $12,6{ }^{\circ} \mathrm{C}$. La precipitación media aproximada es de $567 \mathrm{~mm}$, esta varía hasta 112 mm., entre el mes más seco y el mes más húmedo. Durante el año las temperaturas medias varían en 3,6 ${ }^{\circ} \mathrm{C}$. Se pueden diferenciar 2 estaciones bien marcadas: invierno de mayo a octubre y el verano austral de noviembre a abril, que se caracteriza por ser lluvioso, frío, húmedo y con vientos en las tardes.

\section{2.- INDICADORES DE PRESIÓN}

\section{a) Emisión de gases de efecto invernadero}

El volumen de escombros, desmonte y residuos sólidos vertidos en el entorno del humedal, es aproximadamente, de $90 \mathrm{~m}^{3}$. En Huacarpay la concentración acumulada de $\mathrm{N}_{2} 0$ (óxido nitroso) del 2010 al 2012 fue de $28 \mu \mathrm{g} / \mathrm{m}^{3}$, la concentración acumulada de $\mathrm{H}_{2} \mathrm{~S}$ (sulfuro de hidrógeno) fue de $74,28 \mu \mathrm{g} / \mathrm{m}^{3}$

\section{b) Vías de transporte dentro del humedal}

Presencia de la carretera asfaltada Cusco - Puno, hasta la altura del kilómetro 27. Vías carrozables alrededor del complejo de lagunas del Humedal Lucre-Huacarpay.

\section{c) Turismo}

Elaboración del 'Proyecto de Inversión Pública' (PIP) PIA-Proyecto Circuito Turístico Urpicancha Huacarpay, que en el momento se encuentra en ejecución. 


\section{d) Sobrexplotación de recursos vegetales}

El pastoreo constante en las montañas ha hecho imposible la regeneración espontánea de los bosques y pastos naturales. En algunas zonas la pérdida de suelo es casi completa y el riesgo de erosión y empobrecimiento es permanente.

\section{e) Inundaciones}

El Humedal Lucre-Huacarpay, es susceptible al impacto causado por el desborde de las aguas procedentes del río Huatanay, cuando aumenta su caudal en época de lluvias, mismas que contienen aguas residuales municipales y residuos sólidos, tal como reporta el Instituto Nacional de Defensa Civil (INDECI) en los años 2010 y 2014 (Reporte de situación N. ${ }^{\circ} 118$ 25/01/2014/COEN-INDECI/17:00 horas).

\section{f) Disposición final de los residuos sólidos en la laguna de Huacarpay}

El humedal y la laguna de Huacarpay, que cuenta con cuatro cuerpos de agua que la conforman (Choq'epukyo, Huatón, Pumaorqo y Huáscar), viene registrando cada año, después de las limpiezas periódicas, más de una tonelada de residuos sólidos, lo cual es un tema de preocupación para su conservación.

(http://www.munilucre.gob.pe/actualidad/noticias/44-retiro-de-escombros-desmonte- $\quad$ y-residuos-solidos-del-humedallucre-huacarpay.html).

\section{g) Cambio de los usos de tierras en el ámbito de la laguna de Huacarpay}

Las practicas no sustentables (la conversión de suelos para la agricultura), está afectando directamente al Humedal Lucre-Huacarpay, debido a la pérdida de hábitat para para la diversidad biológica, depredación de especies vegetales, etc.; al introducir prácticas agrícolas y con el uso de fertilizantes, influye en el proceso de eutrofización. (Aragón Romero, 2013). 


\section{h) Vertimiento de aguas residuales en la laguna de Huacarpay}

Existen vertimientos de aguas residuales sin autorización - las cuales no están tratadas-, que se vierten directamente al canal Muyna que posteriormente se dirige a la Laguna Huáscar, que es integrante del Humedal Lucre-Huacarpay, afectando a la calidad del agua. (Autoridad Nacional del Agua - RD N. ${ }^{\circ}$ 117-2013-ANA/AAA XII.UV).

\section{3.- INDICADORES DE ESTADO}

\section{a) Conflictos por el uso del Humedal Lucre}

Los principales conflictos de uso que se han reportado, son por las actividades agrícola y ganadera, así como por el uso de la totora. Las principales causas de conflicto son (Ministerio del Ambiente, 2013):

- Falta de organización, inexistencia de normas locales reconocidas por la población, desobediencia a la autoridad y total falta de respeto.

- Muy poca conciencia del problema que generan los residuos sólidos.

- Deficiente manejo de los recursos naturales.

- Baja identidad cultural y educación.

\section{b) Sistema de cobro por servicios ambientales}

Como todo humedal alto andino, el Humedal Lucre-Huacarpay cumple importantes funciones, como resultado de la interacción de sus componentes bióticos y abióticos, generando beneficios de forma indirecta. El beneficio más importante es: que funciona como filtro natural que limpia las aguas turbias que ingresan a través de los ríos aportantes. Entre los principales servicios ambientales que otorga el humedal están (MINAM, 2013):

- Paisaje y belleza escénica.

- Diversidad biológica y cultural.

- Regulación de carga de acuíferos.

- Retención de nutrientes, sedimentos y contaminantes. 
- Mantenimiento de la cadena trófica.

- Control de la erosión.

- Control de crecidas e inundaciones.

- Exportación de biomasa.

- Estabilización de las condiciones climáticas locales.

- Actividades recreativas y turismo. Actualmente no se realiza ningún cobro o pago por los servicios ambientales que brinda el humedal.

\section{c) Calidad del agua de la laguna Huacarpay}

De acuerdo a la clasificación propuesta por Arrignon (1979), las aguas del humedal son alcalinas y de alta dureza, consideradas como muy productivas.

El agua de la laguna Huatón tiene las siguientes características (Gobierno Regional Cusco, 2016):

- La concentración de oxígeno disuelto disminuye con la profundidad, observándose puntos anóxicos en el bentos.

- La DBO sigue una secuencia inversa a la disminución del oxígeno, incrementándose con la profundidad, debido a la mayor demanda de oxígeno por la materia orgánica del sedimento de fondo.

- Los fosfatos y nitratos presentan concentraciones tendientes al incremento en la columna de agua, desde la superficie hacia la profundidad.

- El amoníaco sigue una secuencia inversa a la concentración del oxígeno; es decir: a menor oxígeno, mayor concentración de amoníaco.

- El plancton predominante está integrado por Navícula, Synedra, Oscillatoria, Tabellaria, Gomphonema, Chroococcus, Anabena y Mycrocystis.

De acuerdo a la información registrada, la laguna Huatón se halla en un estado de mesotrofia con tendencia a la eutrofia, tal aseveración está en función a que el río Lucre aporta, principalmente, fosfatos y la descomposición de la materia orgánica en el cuerpo de agua genera nitratos, los que favorecen al crecimiento estacional de algas y macrófitas. 
En el caso de la laguna Lucre (Pumaorqo), las sustancias en solución provienen de las actividades biológicas y fisicoquímicas existentes en el propio cuerpo de agua y, el aporte de la escorrentía. Su concentración de carbonatos, cloruros y sulfatos es mayor que en la laguna Huatón (aproximadamente en un $13 \%$ ), lo que significa que su pH también es más alcalino. La transparencia es casi completa, por lo tanto, se observa vegetación sumergida (algas), con una concentración del oxígeno disuelto relativamente uniforme, lo que promueve una mayor nitrificación y menor concentración de amoníaco. La posibilidad de eutrofización es menor en comparación con la laguna Huatón.

En los pantanos (incluyendo las lagunas estacionales) la dureza, cloruros, sulfatos, $\mathrm{pH}$ y amoníaco son más altos que en los dos cuerpos de agua lénticos, anteriormente descritos.

\section{d) Oferta hídrica del humedal}

El sistema del Humedal Lucre-Huacarpay, forma parte de la subcuenca del río Huatanay (cuenca Urubamba-Vilcanota), y está conformado por:

- Cuatro lagunas permanentes (Huatón, Pumaorqo, Choq’epuqyo y Huáscar)

- Una laguna temporal (Huacarpay Muina)

- Dos pantanos (Unca y Piscconiyoc)

- Dos ríos (Lucre y Huatanay)

En el ámbito del humedal, hay presencia de aguas subterráneas y superficiales que aportan sus aguas al sistema. Los manantiales identificados son: Santopuqyo con 8,2 1/s de caudal, Choq'epukyo, Mancapuqyo y Misk’iunuyoc.

El volumen de agua del humedal se mantiene casi constante, debido a que sus aguas fluyen en forma perenne por el efluente del noreste, el cual desemboca en el río Huatanay. Las reservas de agua para el humedal ascienden a 1808 428,81 $\mathrm{m}^{3}$. (Gobierno Regional Cusco, 2016). 


\section{e) Ruido}

El ruido es uno de los problemas ambientales más relevantes en el humedal Lucre-Huacarpay y la principal fuente que la produce es el intensivo tráfico vehicular en la vía Cusco - Sicuani y en las vías de comunicación interna (circuito del humedal). El ruido está integrado por dos componentes de igual importancia: una integrante puramente física (el sonido: magnitud física perfectamente definida), y otra integrante de carácter subjetivo, que es la sensación de molestia. Aun cuando no se han hecho evaluaciones, este problema está ocasionando molestias a la fauna silvestre que habita en el humedal.

\section{f) Nivel educativo de los pobladores en el ámbito de la laguna de Huacarpay}

Según el Censo Nacional 2007; XI de Población y VI de Vivienda, un buen porcentaje de la población no tiene ningún nivel educativo $(14,41 \%)$ y más de un tercio $(38,75 \%)$ solo tiene educación primaria.

\begin{tabular}{|l|c|c|}
\hline \multicolumn{1}{|c|}{ Categoría } & Casos & \% \\
\hline Sin nivel & 525 & 14,41 \\
\hline Educación inicial & 101 & 2,77 \\
\hline Primaria & 1412 & 38,75 \\
\hline Secundaria & 1194 & 32,77 \\
\hline Superior no universitaria incompleta & 107 & 2,94 \\
\hline Superior no universitaria completa & 123 & 3,38 \\
\hline Superior universitaria incompleta & 86 & 2,36 \\
\hline Superior universitaria completa & 96 & 2,63 \\
\hline TOTAL & $\mathbf{3 6 4 4}$ & $\mathbf{1 0 0 , 0 0}$ \\
\hline
\end{tabular}

Respecto a la tasa de analfabetismo, en la zona de influencia del humedal la tasa es de $19,34 \%$, cifra mucho mayor que el promedio nacional (13,13\%), según el Censo de Población y Vivienda de 2007.

\section{g) Ingresos reportados por actividades económicas en el humedal}

La mayor parte de la población $(71,88 \%)$ se dedica a la agricultura y ganadería extensiva (vacuna y ovina), y el $30 \%$ aproximadamente de la producción agrícola, se destina al autoconsumo; en este sentido, las familias se caracterizan por poseer una economía de 
subsistencia, con bajos ingresos económicos que no les permite disponer de alimentos básicos.

\begin{tabular}{|l|c|c|}
\hline \multicolumn{1}{|c|}{ CATEGORÍA } & CASOS & \% \\
\hline Agricultura, ganadería & 116 & 71,88 \\
\hline Explotación de minas y canteras & 5 & 3,13 \\
\hline Industrias manufactureras & 2 & 1,25 \\
\hline Construcción & 2 & 1,25 \\
\hline Comercio por menor & 18 & 11,25 \\
\hline Hoteles y restaurantes & 4 & 2,50 \\
\hline Transporte y comunicaciones & 10 & 6,25 \\
\hline Enseñanza & 1 & 0,63 \\
\hline Servicios sociales y de salud & 1 & 0,63 \\
\hline Otras actividades & 1 & 0,63 \\
\hline Hogares privados y servicios domésticos & 1 & 0,63 \\
\hline TOTAL & $\mathbf{1 6 1}$ & $\mathbf{1 0 0 , 0 0}$ \\
\hline
\end{tabular}

Fuente: Censo Nacional 2007: XI de Población y VI de Vivienda

\section{h) Saneamiento básico}

Respecto al abastecimiento de agua, menos del $30 \%$ de las viviendas cuentan con acceso a una red de agua potable, mientras que cerca del $56 \%$ de la población accede al agua fuera de la vivienda. Las fuentes de agua no son nada seguras y exponen de forma considerable la salud de la población.

\begin{tabular}{|l|c|c|}
\hline CATEGORÍAS & $\mathbf{2 0 0 7}$ & \% \\
\hline Red Publica Dentro de la Vivienda & 258 & 26,03 \\
\hline Red Publica Fuera de la Vivienda & 553 & 55,80 \\
\hline Pilón Publico & 19 & 1,92 \\
\hline Pozo & 7 & 0,71 \\
\hline Camión / Cisterna & 6 & 0,61 \\
\hline Rio, Acequia, Manantial & 99 & 9,99 \\
\hline Vecino & 36 & 3,63 \\
\hline Otro & 13 & 1,31 \\
\hline TOTAL & $\mathbf{9 9 1}$ & $\mathbf{1 0 0} \%$ \\
\hline
\end{tabular}

Fuente: Censo Nacional 2007: XI de Población y VI de Vivienda 
Asimismo, el 73,03 \% de la población no cuenta con servicio de desagüe.

\section{4.- INDICADORES DE IMPACTO}

\section{a) Pérdida de biodiversidad}

La degradación de los humedales es un fenómeno presente a nivel mundial, tiene especial connotación en los andes al ser bofedales y totorales: ecosistemas de gran fragilidad. Los totorales del Humedal Lucre-Huacarpay están siendo afectados por el sobrepastoreo y la ampliación de la frontera agrícola; la construcción de reservorios, diques y canales que alteran los ciclos naturales de especies biológicas; la carretera que circunda al humedal que ocasiona la muerte de animales arrollados; la extracción insostenible de recursos —como la totora-, causan la pérdida de la biodiversidad. (Aragón Romero, 2013).

Refugio natural de aproximadamente 108 especies de aves durante la mayor parte del año. Especies nativas de diminutos peces como el 'ch'iñi challwa', que — según algunos pobladores- estarían a punto de desaparecer.

\section{b) Disminución de la calidad del agua del humedal}

En todo el mundo (y el Cusco no es la excepción), los seres humanos estamos perturbando las fluctuaciones naturales de los niveles de agua; esta alteración hidrológica es una de las tres principales causas de daños a la fauna acuática. La naturaleza de la alteración depende de si se construyen reservorios río arriba o represas río abajo o canales de encauzamiento; un efecto casi universal es la reducción de la inundación en lluvias y la subsiguiente disminución del área del humedal, permitiendo su conversión en suelos agrícolas.

Los efectos de la construcción de reservorios en las cabeceras de cuencas, dependen del propósito: si el objetivo es proveer pulsos de agua para demanda de riego y energía, las fluctuaciones pueden aumentar... y pueden disminuir si el objetivo es almacenar agua; por tal razón, deben evaluarse sus efectos caso por caso. En el tema de la construcción de represas, la estabilización de los niveles de agua destruye los ciclos de inundaciones 
necesarias para la existencia de los humedales río abajo este efecto, también, es logrado cuando se encauzan los ríos. El patrón general es, entonces, que estas construcciones reducen las inundaciones, lo que a su vez, lleva a que los humedales disminuyan y que sean invadidos por vegetación terrestre o, en nuestros tiempos, por zonas agrícolas.

\section{c) Reducción del espejo de agua}

En el Humedal Lucre-Huacarpay, los espejos de agua tienen superficies cambiantes en extensión durante el año, sin embargo, es notoria la reducción del espejo de agua debido, principalmente, al proceso de eutrofización y avance de las áreas de totora.

\section{d) Eutrofización}

Una consecuencia de la civilización es la erosión de suelos y el aumento de la fertilización en la agricultura, como resultado: han aumentado el contenido de nitratos y fosfatos en el agua de lluvia y escorrentía.

Estos nutrimentos se acumulan en los humedales con cuatro consecuencias generales: (1) los gradientes de fertilidad son perturbados, llevando a una disminución de la diversidad de especies vegetales; (2) las plantas dominantes se vuelven más apetecibles, aumentando poblaciones de herbívoros; (3) en aguas someras, el aumento de algas flotantes puede matar a las plantas emergentes características de los humedales; y (4) cuando se descomponen estas algas y plantas emergentes, el oxígeno es consumido, lo que lleva a condiciones anóxicas que matan a los peces. Estos dos últimos factores tienen factores negativos en la producción piscícola y llegan a destruir la diversidad biológica de los humedales.

En tanto, el aumento de erosión y fertilizantes desde zonas agrícolas ha aumentado gradualmente la eutrofización en humedales, los efectos más dramáticos provienen de las mismas ciudades de donde vienen cargas más fuertes de erosión por construcciones y desmontes y, especialmente, los desagües. Esto puede notarse ya en la desembocadura del río Lucre en Huacarpay, además, que la contaminación del Huatanay ha tenido graves efectos para el valle del Vilcanota. 
El incremento de las poblaciones de algas y de la 'lenteja de agua' (Lemna gibba) en los humedales eutrofizados, es una amenaza seria al avasallar a plantas emergentes, como la totora y la matara, disminuyen los niveles de oxígeno en el agua. Estas plantas acuáticas emergentes son importantes, debido a que son bombas que quitan el exceso de nutrimentos en el sedimento y lo devuelven hacia aguas abiertas, por lo que son importantes para restaurar humedales.

\section{5.- INDICADORES DE RESPUESTA}

\section{a) Participación colectiva en actividades para la conservación del humedal}

Gestión del humedal a cargo de la Municipalidad de Lucre, con apoyo de la Autoridad Administrativa RAMSAR, el Gobierno Regional del Cusco y la población.

Actividades de sensibilización ambiental y comunicación a los pobladores; vigilancia y organización para cuidar el humedal.

\section{b) Planificación para la conservación del humedal}

Se logró un plan de gestión participativo con los actores implicados; proyecto de gestión y ecoturismo para la conservación del humedal.

\section{c) Análisis de la calidad del agua de la laguna de Huacarpay}

De acuerdo a los últimos estudios realizados (Acuña, 2003) en el Humedal Lucre-Huacarpay, el índice de calidad del agua fluctúa entre 26,95 y 59,39, que según el índice de calidad de agua dado por Mitchell, et al. (1991), corresponden a aguas de 'calidad mala' en la zona del bentos, y aguas de 'calidad mediana' en la superficie. Para el río Lucre — principal afluente del humedal—, se determinó un índice de calidad entre 58,94 y 66,46, valores que corresponderían a aguas de 'calidad mediana'.

Se han realizado muchos estudios referidos a las características fisicoquímicas de las aguas del humedal, cuyos datos han ido registrando incrementos (temperatura, DBO, nitratos, fosfatos), y disminuciones (oxígeno disuelto), a través de los años hasta la fecha, lo cual 
demuestra que el proceso de eutrofización está en aumento, disminuyendo de manera drástica la calidad del agua, lo que hace suponer que en un futuro no muy lejano estas aguas dejarán de ser aptas para la mayor parte de organismos que lo habitan, por lo que urge tomar medidas al respecto.

Se necesita una ordenanza municipal para la regulación de emisión de residuos sólidos y líquidos que son arrojados y vertidos al área del humedal.

\section{d) Prácticas de control de erosión en la Laguna de Huacarpay}

Reforestación en la cuenca del humedal con especies nativas, principalmente molle (Schinus molle) y Huaranhuay (Tecoma stans).

\section{e) Reconocimiento del Humedal Lucre-Huacarpay como sitio Ramsar}

El dos de febrero de 1971 se firmó el Convenio sobre los Humedales, a orillas del Mar Caspio, en la ciudad iraní de Ramsar. Con él se creó una lista de las zonas húmedas de importancia internacional compuesta por 2065 humedales de 168 países, entre los que se incluyen 13 reconocidos en el Perú, de los cuales tenemos: los Bofedales y Laguna de Salinas, el Complejo de humedales del Abanico del río Pastaza, el Humedal Lucre-Huacarpay, el Lago Titicaca (compartido con Bolivia), la Laguna del Indio-Dique de los Españoles, las Lagunas las Arrebiatadas, los Manglares de San Pedro de Vice, el Pacaya-Samiria, Paracas, la Reserva Nacional de Junín, el Santuario Nacional Lagunas de Mejía, el Santuario Nacional Los Manglares de Tumbes y la Zona Reservada los Pantanos de Villa.

La Convención Ramsar entró en vigor en nuestro país en 1975, el Perú se incorporó como miembro activo en 1991, correspondiéndole a la Dirección General de Áreas Protegidas del INRENA desempeñarse como autoridad administrativa ante la Convención. El Humedal Lucre-Huacarpay, fue designado como Sitio Ramsar, el 23 de septiembre del 2006.

\section{f) Tecnologías para la conservación del humedal}

Diseño y construcción de infraestructuras ecoturísticas (observatorios y miradores), construcción de muro de contención en el sector noroeste del humedal; diseño de un sistema 
de saneamiento de aguas residuales; distribución de ollas solares y capacitación para su uso como alternativa al uso de leña. Se realizó mantenimiento y reforestación de la plantación de árboles alrededor de las lagunas, siendo la superficie reforestada de 24,94 ha., con 320000 árboles plantados, entre ellos: molle (Schinus molle), chachacomo (Escallonia resinosa), q'euña (Polylepis racemosa).

\section{g) Participación de la población para la conservación del humedal}

En diciembre de 2005, se realizaron cursos de capacitación en forestación y reforestación de la Laguna de Huacarpay, y se han capacitado a 101 personas. A finales de noviembre de 2006, se inició la plantación de 27000 árboles en Lucre, sembrándose 3 especies arbóreas nativas (q'euña, chachacomo y molle). Al 2006, se realizó una cerca protectora de 1750 metros lineales. Al 2010 se realizaron actividades de reforestación en 6,5 ha, con 12000 plantas de molle (Schinus molle) y huaranhuay (Tecoma sambucifolia), mantenimiento de las plantaciones, limpieza, desbroce, riego y reposición.

\section{h) Identificación de biota (aves)}

Se realizó una primera lista de aves del humedal para facilitar su observación. De acuerdo a información proporcionada por el Comité Técnico Interinstitucional y Concertado del Humedal de Huacarpay y Microcuenca de Lucre, en un informe difundido por el Centro Guamán Poma de Ayala; se sabe que este sistema natural es fuente de alimento y refugio de, aproximadamente, 108 especies de aves durante la mayor parte del año, algunas son endémicas y otras se hallan en peligro de extinción.

\section{i) Educación ambiental}

Se propicia la formación de las mujeres en el uso de cocinas solares. Durante el primer semestre de 2005, se realizaron actividades de capacitación de la población local, participando un total 1414 personas en 35 talleres y charlas sobre ecoturismo.

Finalmente, luego de seleccionados los indicadores se procedió, a la obtención de información por medio de revisión bibliográfica. Esta información ingresó al modelo 
FMPEIR como dato. Luego se procedió a la validación de las variables mediante la fundamentación conceptual de cada una de ellas en su factor de análisis.

Una vez obtenidos los indicadores por factor de análisis, se empleó el 'diagrama radial de la rosa de los vientos', donde cada eje corresponde a un indicador y está dividido en la escala de evaluación de 1 a 5 para el cálculo del ISHLH (ver tabla 1).

Con esta metodología se obtuvo un área síntesis, que representa los resultados de los 33 indicadores FMPEIR definidos para el ISHLH; el porcentaje de esta 'área síntesis', respecto al 'área ideal', la cual ocurre cuando todos los indicadores han sido calificados con un valor que va del uno al cinco, corresponde al Índice de Sostenibilidad del Humedal Lucre Huacarpay (ISHLH).

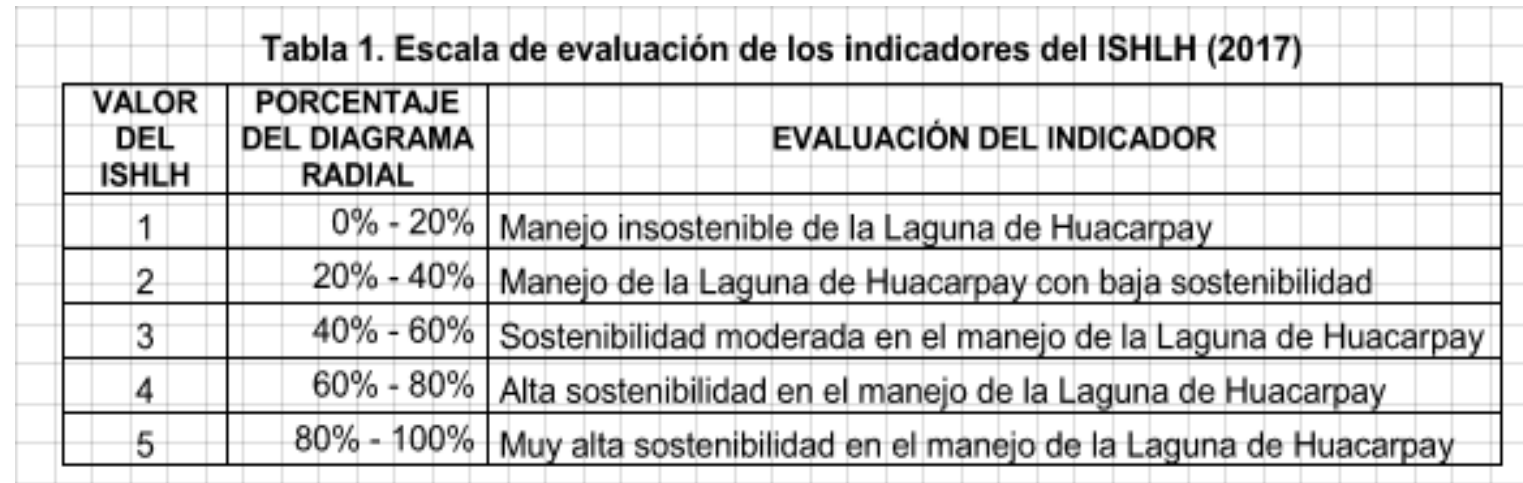

\section{RESULTADOS}

Como se observa en la figura 1, las fuerzas motrices representan el $12 \%$ de los indicadores encontrados en el estudio. De estos, los indicadores que más resaltan son: el 'crecimiento demográfico' y el 'incremento de las actividades económicas', con una puntuación de 3 y 2 respectivamente.

Por otro lado, los indicadores de presión hacen un $24 \%$ del total, con los indicadores 'emisión de gases de efecto invernadero' y las 'actividades turísticas alrededor de la laguna de Huacarpay', como los indicadores más resaltantes, con una puntuación de 4 cada uno. En cuanto a los indicadores de estado, se tiene que forman parte del $24 \%$ del total de indicadores encontrados. 
De estos, los más resaltantes son la 'calidad del agua' y los 'conflictos por el uso de la laguna de Huacarpay’, ambos con una puntuación de 3. En referencia a los indicadores de impacto, se tiene el $12 \%$ del porcentaje total de los indicadores, tales como 'pérdida de biodiversidad' y 'disminución de la calidad del agua del humedal', con una puntuación de 3 cada uno. Para dar con los indicadores de respuesta, se obtuvo dos que tienen mayor puntuación, que son: el 'reconocimiento del humedal Lucre-Huacarpay como sitio Ramsar' con una puntuación de 5 y, el indicador 'tecnologías para el uso del humedal', con una puntuación de 4. Ambos dentro del $28 \%$ del total de indicadores encontrados en toda la metodología FMPEIR.

Los aspectos a mejorar están relacionados con el sistema de la disposición final de los residuos, tema relacionado directamente con la gestión ambiental integral de los recursos de la microcuenca del río Lucre, la cual es deficiente y genera problemas para la creación y aplicación de estrategias para la conservación del humedal. Por otro lado, estas pueden mejorarse a través de políticas públicas ejecutadas por la administración municipal, enfocadas a satisfacer las necesidades básicas, aumentar el nivel de vida de los pobladores y lograr el desarrollo sostenido y sostenible.

Otro punto importante a fortalecer hace referencia a los vertimientos contaminantes que se descargan a las fuentes hídricas, que aunque no obtienen la más alta calificación, generan un aumento en la presión antropogénica sobre los recursos del humedal. En cuanto al desarrollo de actividades para la conservación del recurso hídrico, es importante mejorar la participación de instancias públicas en la zona, puesto que, durante los últimos años no se han desarrollado acciones con tal fin, en su defecto, ha sido la comunidad la que se ha organizado. 


\section{FIGURA 1}

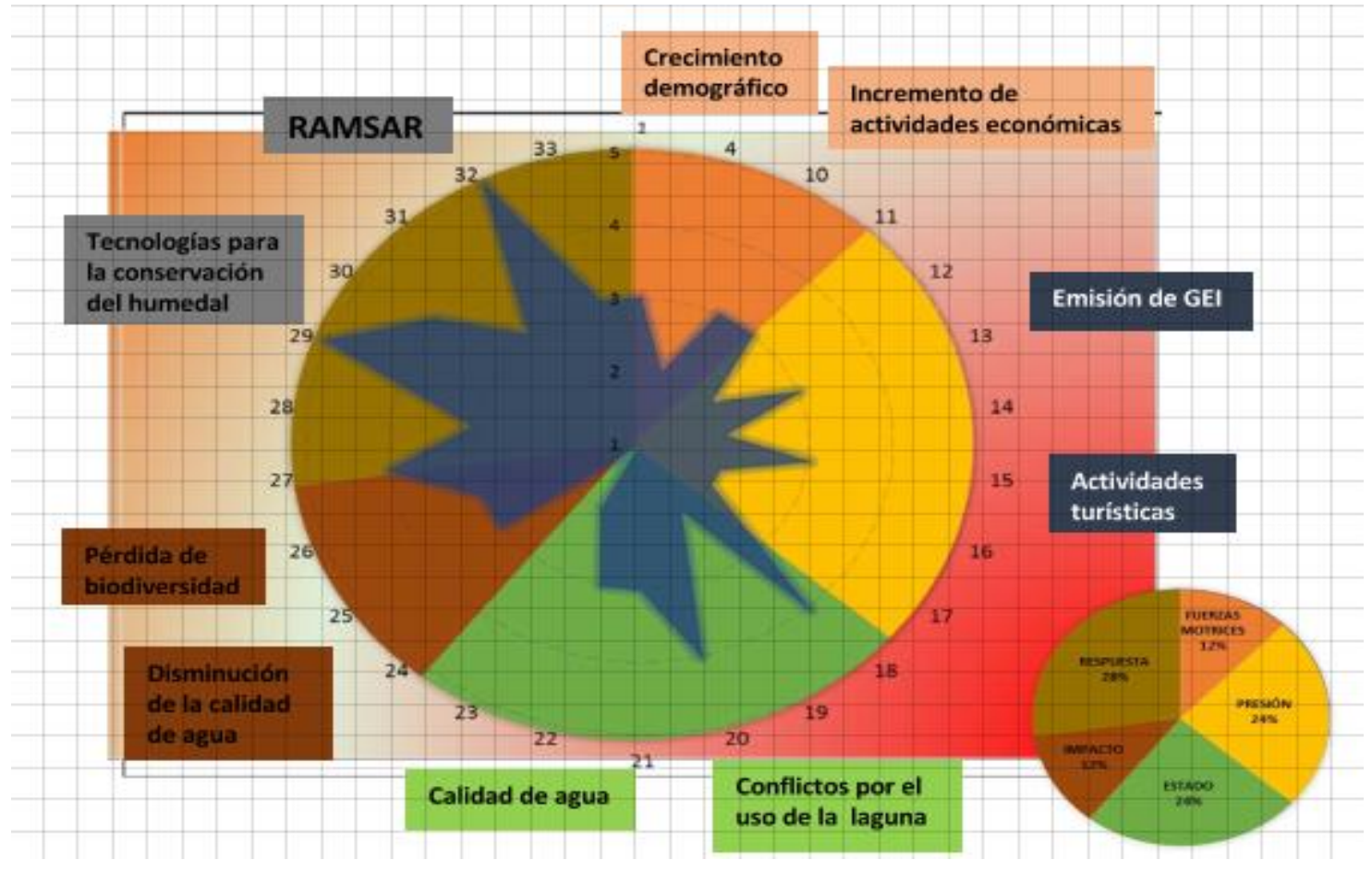

\section{DISCUSIÓN}

La utilización de la metodología FMPEIR, es óptima para la elaboración de políticas públicas referentes a la interacción de las respuestas frente a los otros componentes de la metodología que conllevan a la determinación de indicadores ambientales y elaborar actividades de sostenibilidad en temática ambiental. El crecimiento demográfico y el desarrollo económico, son considerados como fuerzas motrices ubicuas del cambio ambiental e incluyen, aspectos particulares que ejercen presión: energía, transporte, urbanización y globalización. Las respuestas políticas deben concentrarse en la reducción de las presiones y así enfrentar los efectos.

Los índices, son herramientas creadas para obtener un diagnóstico con mirada sintética de la realidad; y en esa medida, el Índice de Sostenibilidad del Humedal Lucre-Huacarpay (ISHLH), ha mostrado una representación sintética de lo que acontece en el Humedal LucreHuacarpay.

A pesar de que el ISHLH desarrollado en la investigación constituye un importante instrumento para el análisis y la toma de decisiones, es indispensable continuar trabajando 
en la creación de nuevas herramientas que no solo contemplen el recurso hídrico, sino que evalúe la sostenibilidad de los sistemas productivos, como el humedal completo, con mirada más amplia e involucre otros aspectos como: la biodiversidad y la adaptación al cambio climático.

\section{CONCLUSIONES}

El análisis de los resultados de la aplicación del índice mostró una sostenibilidad media-baja del humedal.

No obstante, es importante fomentar la participación de instituciones públicas y privadas en proyectos que permitan crear mecanismos y promuevan la economía y el desarrollo de la región.

Con la aplicación y evaluación del ISHLH, se pueden diseñar estrategias de sostenibilidad en torno al complejo del humedal, con el objetivo de mitigar los desequilibrios ambientales presentes, la pérdida de la calidad y cantidad de agua, el manejo inadecuado del ecosistema, la degradación de los suelos, conflictos sociales, déficit de política pública que promueva el desarrollo de las zonas rurales, entre otras; lo cual hace necesario plantear nuevas acciones hacia el desarrollo sostenible y competitivo del complejo del humedal como un ecosistema.

\section{AGRADECIMIENTOS}

Este estudio ha sido posible por el apoyo e interés en promover la investigación en la zona de estudio, encabezado por la Dra. Arq. Shirley Chilet Cama; por su orientación y la aplicación práctica y tuvo la paciencia de analizar y discutir los contenidos del estudio, brindando valiosas apreciaciones que han enriquecido el artículo; asimismo, a todos los integrantes del grupo por la colaboración y participación activa en el desarrollo de la presente investigación.

\section{Contribuciones de autoría}

YA, DA y GG generaron la idea de investigación y el diseño del estudio, realizaron la recolección y análisis de datos, EC elaboró el manuscrito y WC y PS aportaron de manera crítica a su contenido, todos los autores aprobaron la versión final a publicar.

\section{Conflictos de interés}

Los autores declaran no tener conflictos de interés en la publicación de este artículo. 


\section{REFERENCIAS BIBLIOGRÁFICAS}

1. Astier, M. \& Masera, O., (1997). Documento de trabajo 17. Metodología para la Evaluación de Sistemas de Manejo incorporando Indicadores de Sostenibilidad (MESMIS). México: Grupo interdisciplinario de Tecnología Rural Apropiada.

2. Castro, M., (2002). Indicadores de Desarrollo Sostenible Urbano. Una aplicación para Andalucía. [En línea]. Doctorado en Economía, Departamento de Economía Aplicada, Estadística y Econometría, Universidad de Málaga, Málaga, enero 30 2017. Disponible en Internet: http://www.eumed.net/tesis/jmc/index.htm

3. Chirino, E., Abad, J. \& Bellot, (2008). Uso de indicadores de Presión-Estado-Respuesta en el diagnóstico de la comarca de la Marina Baixa, Ecosistemas, 17 (1), 107-114.

4. Escobar, L., (2004). Construcción de índices de calidad ambiental urbana: Un modelo general y aplicación para Cali, Colombia. Trabajo de Investigación Universidad de Alcalá, España.

5. Gobierno Regional Cusco, (2016). PIP Recuperación del ecosistema del sitio Ramsar Humedal Lucre Huacarpay. Cusco, Perú.

6. Instituto Nacional de Estadística e Informática- INEI, (2007). Censo Nacional 2007: XI de Población y VI de Vivienda. Lima, Perú.

7. Instituto Nacional de Ecología, INE, (1997). Avances en el desarrollo de indicadores para la evaluación del desempeño ambiental en México. [En línea]. INE, México, enero 23 2011. Disponible en Internet: http://www2.ine.gob.mx/publicaciones/libros/126/marco.html

8. Ministerio del Ambiente, (2013). Plan de Gestión Participativo del Humedal Lucre- Huacarpay. Cusco, Perú.

9. Organización para la Cooperación y el Desarrollo Económico, OCDE/OECD, (1993). OECD Core Set of Indicators for Environmental Performance Reviews, Environment Monographs, N. ${ }^{\circ} 83,1$ 1-39.

10. Pérez, M., (2003). Marco conceptual y metodológico para la construcción de indicadores ambientales. Nicaragua: Sistema Nacional de Información Ambiental, SINIA, 14p.

11. Reyes, A., (2007). Metodología para la integración social del conocimiento en el marco de las buenas prácticas agrícolas del sector hortifrutícola en cinco municipios del valle del cauca. Tesis de Maestría. Universidad del Valle, Colombia.

12. Segnestam, L., (2002). Indicators of environment and sustainable development: Theories and practical experience. Environmental Economics Series. Paper N. ${ }^{\circ} 89$. The World Bank Environment Department.

13. Aragón Romero, J. I. (2013). Los humedales de la Región Cusco. Cusco: Gobierno Regional del Cusco.

14. UNESCO. (1971). Convención Relativa a los Humedales de Importancia Internacional Especialmente como Hábitat de Aves Acuáticas. Ramsar - Irán: ONU. Recuperado el 18 de setiembre de 2017, de

http://www.ramsar.org/sites/default/files/documents/library/current_convention_s.pdf 